\title{
Numerical Stimulation of Hydrodynamic Performance for Twin-skeg Buoy Tender in Shallow Water
}

\author{
Wang Yujuan ${ }^{1, a}$, Huang Sheng ${ }^{1}$, Feng Feng ${ }^{1}$, Liu Yu ${ }^{1, b}$, \\ Li Xiaojiao ${ }^{1}$, Wang Gang $^{2}$ \\ ${ }^{1}$ College of Shipbuilding Engineering, Harbin Engineering University, Harbin, 150001, China \\ ${ }^{2}$ China Petroleum Liaohe Equipment Company, Panjin,124000, China \\ ayj020111@163.com, bliuyu1984@hotmail.com
}

Key words: Twin-skeg buoy tender, shallow-water resistance, FLUENT, numerical stimulation

\begin{abstract}
In this paper, we obtain the detailed and precise data of the resistance performance in shallow water for the twin-skeg buoy tender in the different shallow-water conditions by the numerical stimulation of the resistance characteristic with the assistance of the commercial software FLUENT. Otherwise, the above obtained data are used to compare with the experimental results of the corresponding model in the towing resistance experiment. FLUENT is indeed competent and reliable for the calculation of the ship resistance.
\end{abstract}

\section{Introduction}

Viscosity can offer viscous resistance, which is generated by the viscous effect. As the definition, we can distinct the viscous resistance into two forms, the frictional resistance, and the viscous pressure resistance (VPR). Generally, the frictional resistance takes absolutely major account for the low-speed vessel, with the percentage from $70 \%$ to $80 \%$. But for the high-speed vessel, the frictional part decreases to $40 \% \sim 50 \%$ and the VPR declines to $10 \%$ approximately. So we can know, from above, that the viscous resistance takes a relatively higher portion within the total resistance.

\section{Calculation model}

\section{Model Construction}

The model of the Heilongjiang twin-skeg buoy tender, as the calculated object in this paper, illustrated in Fig.1, is scaled with the designed waterline $4.0 \mathrm{~m}$, the moulded breadth $0.914 \mathrm{~m}$, the still-water draft $0.143 \mathrm{~m}$, the overall wetted surface $2.682 \mathrm{~m}^{2}$, and the overall displacement $0.285 \mathrm{~m}^{3}$. According to the relevant experience ${ }^{[8]}$, the computational domain is a rectangle. We use the half hull to calculate. Then, $2.5 \leqslant x / L \leqslant 1.5,0 \leqslant y \leqslant L$. are confirmed as the computational domain, where $x$ is the length beyond the bow along the $X$ axis, $y$ is the distance from the moulded breadth to the edge of the domain along the $Y$ axis, and $L$ is the length of the waterline of the model. In order to guarantee the channel depth and the air height, $x$ and $y$ need to vary for the calculation of different twin-skeg buoy tenders.

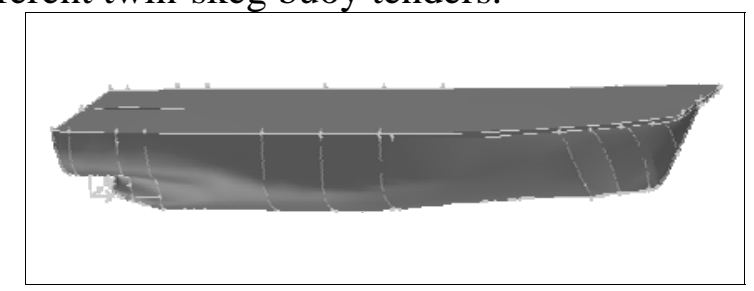

Fig.1 Twin-skeg Ship’s model

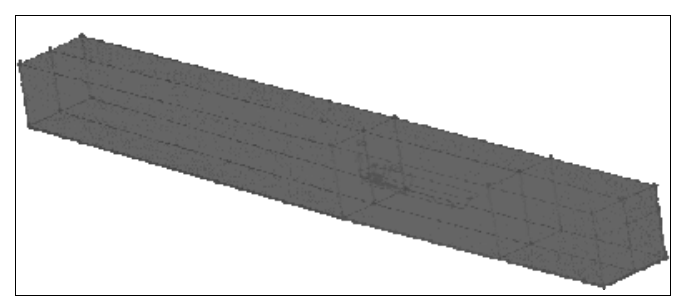

Fig.2 Twin-skeg Ship’s model’s grid partition

Grid Partition

For the instance, in the infinite-depth condition, the mesh number of the entire domain totals up to $1,675,808$, where the mesh number of the vessel hull amounts for 377,502 . By the examinational function supplied by the Gambit, the test towards the grid quality shows that the maximum 
asymmetry of this model is 0.77 , which is not greater than $0.97,0.97$ which is defined as the maximum asymmetry that should not be exceeded. Otherwise, the asymmetry of most meshes is limited from 0.1 to 0.6 , and there is merely $4.6 \%$ meshes with the asymmetry greater than 0.6 . Therefore, we can say that this domain has an excellent grid partition that can ensure the calculation precision of CFD. The grid partition is showed in Fig.2.

1.3 Settings for Boundary Conditions

We can see the settings in Table.1, as following

Table 1 Boundary Conditions

\begin{tabular}{|c|c|c|c|c|c|c|}
\hline Position & Water-Inlet & Outlet & Symmetry & Surface & Water & Others \\
\hline $\begin{array}{l}\text { Boundary } \\
\text { Condition }\end{array}$ & VELOCITY-INLET & $\begin{array}{c}\text { FREE } \\
\text { OUTFLOW }\end{array}$ & SYMMETRY & WALL & FLUID & WALL \\
\hline
\end{tabular}

1.4 Numerical Stimulation \& Solution

Table 2(a) Model Speeds and Corresponding Real Speeds for the IDENTICAL Vessel

\begin{tabular}{c|c|c|c|c|c|c|c}
\hline Model Speed(m/s) & 1.05 & 1.26 & 1.47 & 1.68 & 1.89 & 2.1 & 2.31 \\
\hline Real Speed(km/h) & 10 & 12 & 14 & 16 & 18 & 20 & 22 \\
\hline \multicolumn{2}{l}{ Table 2(b) Model Speeds and Corresponding Real Speeds for DIFFERENT Vessels } \\
\hline \multicolumn{2}{l|}{ Model Speed(m/s) } & 1.05 & 1.26 & 2.1 & 2.31 \\
\hline
\end{tabular}

Firstly, in the infinite-depth condition, the resistance from the IDENTICAL vessel shall be stimulated for calculation in various model speeds, showed in Table 2(a). Besides, the resistance from DIFFERENT vessels, cruising in different depth conditions, will be stimulated in the same speeds, showed in Table 2(b), with the model depths, showed in Table 2(c).

\section{Result Analysis}

Feasibility demonstration of FLUENT

With the purpose to definite the error range, the FLUENT feasibility is demonstrated firstly by stimulation of the model resistance in the infinite-depth condition, which concludes such following results, that is illustrated in the below figures where, for the instance of the model speed $1.47 \mathrm{~m} / \mathrm{s}$, the nephograms of the upper pressure, of the cruising speed, and of the fluid field distribution are clearly displayed.

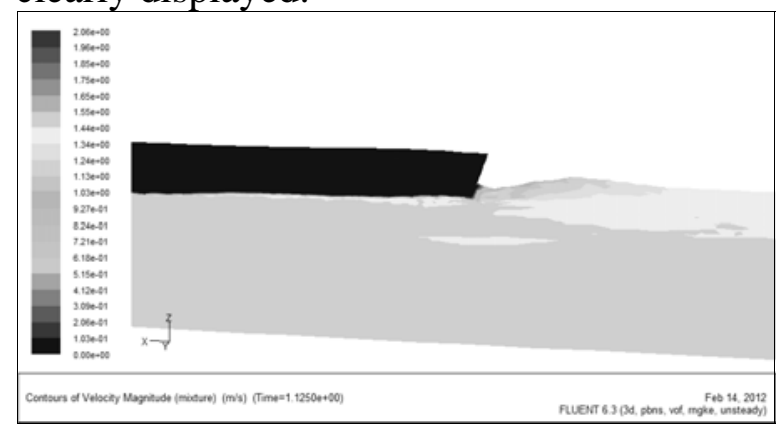

Fig.3 The distribution of free surface

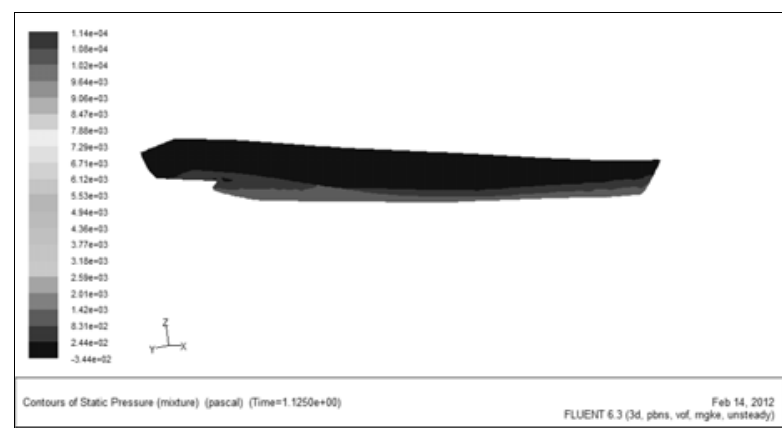

Fig.4 The distribution of the pressure 


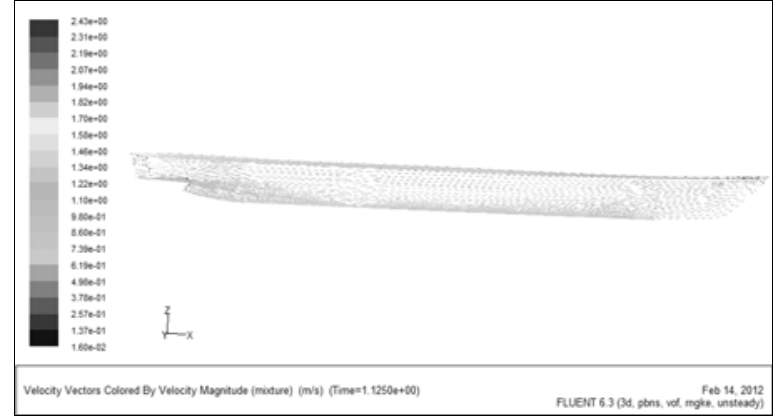

Fig.5 The distribution of velocity

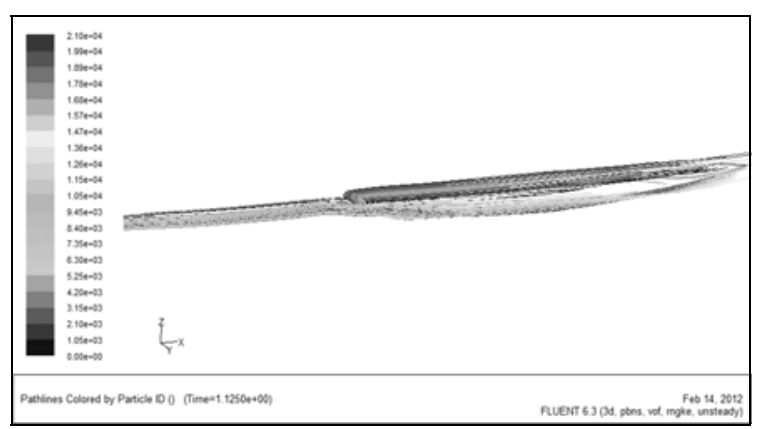

Fig.6 The flow distribution

We can figure out the errors, displayed in Table 3, by the comparison between the stimulation results and the experimental results, illustrated in Fig.7. The feasibility and the validity of FLUENT are demonstrated.

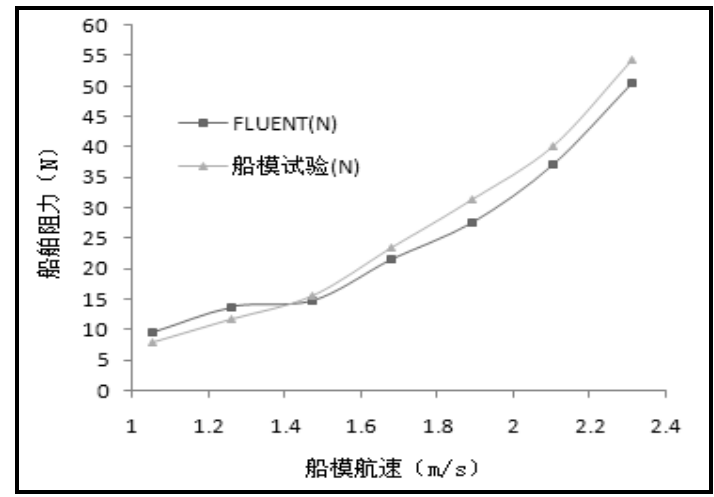

Fig.7 The compared result of model resistance

Likewise, by such the comparison, we find that, the error reaches up to $15 \%$, in the low-speed condition, but becomes lower to approximately $7 \%$ in high-speed condition. Therefore, the FLUENT users need to be aware of the existence of the precision range and the error range, especially in the low-speed condition. Actually, it is feasible to calculate the viscous resistance of the twin-skeg buoy tender, because of the acceptable error range from $10 \%$ to $15 \%$. Assuredly, FLUENT is quite suitable for the high-speed condition.

Research on the shallow-water performance of the twin-skeg buoy tender

The resistances in different depths need to be stimulated in the fixed speeds. In this paper, we adopt $\mathrm{V}=1.05 \mathrm{~m} / \mathrm{s}, \mathrm{V}=1.47 \mathrm{~m} / \mathrm{s}, \mathrm{V}=2.1 \mathrm{~m} / \mathrm{s}$, and $\mathrm{V}=2.31 \mathrm{~m} / \mathrm{s}$ as the model speeds, and calculate respectively the model resistance in the depths $0.214 \mathrm{~m}, 0.286 \mathrm{~m}, 0.357 \mathrm{~m}, 0.429 \mathrm{~m}, 0.571 \mathrm{~m}, 0.714$ $\mathrm{m}$, and $0.857 \mathrm{~m}$. By the numerical stimulation and calculation of the vessel resistance portion in different depths and in every fixed speed with the assistance of FLUENT, and also by the analysis of the affect on the different resistance portion from the shallow-water effect, the change tendency can be summarized to further resolve the shallow-water performance of the twin-skeg buoy tender.

2.2.1 Viscous resistance in shallow water

Table 3 Viscous resistance under different channel depth (MS: Model Speed and MD: Model Depth)

\begin{tabular}{c|c|c|c|c}
\hline $\mathrm{MS}(\mathrm{m} / \mathrm{s})$ & 1.05 & 1.47 & 2.1 & 2.31 \\
$\mathrm{MD}(\mathrm{m})$ & & & & \\
\hline 0.286 & 6.355 & 11.252 & 22.252 & 28.717 \\
\hline 0.429 & 6.011 & 11.243 & 21.571 & 28.383 \\
\hline 0.571 & 5.998 & 11.068 & 19.121 & 27.111 \\
\hline 0.714 & 5.531 & 9.157 & 17.754 & 26.843 \\
\hline 0.857 & 5.035 & 7.053 & 16.443 & 25.993 \\
\hline
\end{tabular}

In the shallow-water condition, the vessel viscous resistance will be directly affected by the channel depth, i.e. the shallower the channel depth is, for the identical vessel under the same speed, the greater the viscous resistance will be. The reason is that, the limited depth can increase the speed of the return flow, so that, part of fluid flow is squeezed forwards along the sides, which makes the speed much greater than that in deep-water condition, and further, the speed increase 
leads to the pressure decrease, that results to the draft increase and the wetted-surface increase, which eventually makes the increase of the viscous resistance of the vessel.

2.2.2 Shallow-water affection on the wave-making resistance

Table 4 Wave resistance under different channel depth (MS: Model Speed and MD: Model Depth)

\begin{tabular}{c|c|c|c|c}
\hline $\mathrm{MS}(\mathrm{m} / \mathrm{s})$ & 1.05 & 1.47 & 2.1 & 2.31 \\
\hline 0.286 & 10.106 & 22.331 & 34.622 & 39.739 \\
\hline 0.429 & 9.675 & 15.995 & 32.018 & 36.202 \\
\hline 0.571 & 8.188 & 15.853 & 29.442 & 35.045 \\
\hline 0.714 & 8.351 & 15.197 & 28.231 & 29.175 \\
\hline 0.857 & 4.98 & 11.638 & 27.269 & 27.43 \\
\hline
\end{tabular}

We can find, in Table 4, when the vessel is cruising in the shallow-water channel, the shallower, the greater the wave-making resistance is. The reason is that, the wave covering area is increased due to the shallow water, which raises the wave height; meanwhile decrease the wave speed, as a response, the wave-making resistance increases obviously. Simultaneously, the wave-making resistance will become relatively greater along with the increase of the speed within a certain speed range.

2.2.3 Shallow-water affection on the model

As analyzed above, we know that, the vessel suffers significant shallow-water effects in different depths, or various speeds in the shallow-water conditions, which lead to the resistance changes of the models. The total resistances of the model in different channel depths are showed in Table 5.

Table 5 Total resistance under different channel depth (MS: Model Speed and MD: Model Depth)

\begin{tabular}{c|c|c|c|c}
\hline $\mathrm{MS}(\mathrm{m} / \mathrm{s})$ & 1.05 & 1.47 & 2.1 & 2.31 \\
\hline $\mathrm{MD}(\mathrm{m})$ & 16.461 & 33.583 & 56.874 & 68.456 \\
\hline 0.286 & 15.686 & 27.238 & 53.589 & 64.585 \\
\hline 0.429 & 14.186 & 26.921 & 48.563 & 62.156 \\
\hline 0.571 & 13.882 & 24.354 & 45.985 & 56.018 \\
\hline 0.714 & 10.015 & 18.691 & 43.712 & 53.423 \\
\hline 0.857 &
\end{tabular}

The increase percentages of the total resistance for this twin-skeg buoy tender in the shallow water condition are distributed in Fig.8.

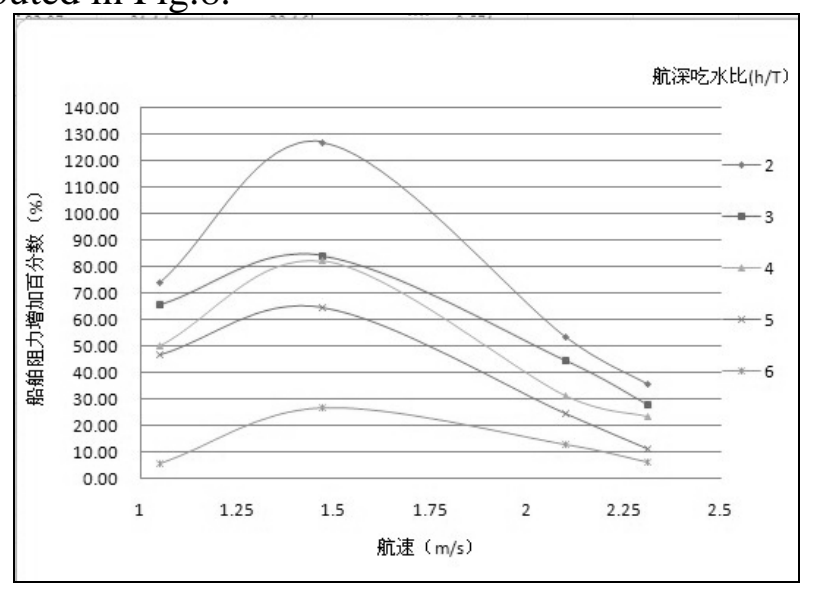

Fig.8 The distribution of the increasing percentage

As we can see in Table 5, in a fixed range of the speeds, the shallow-water effect tends to be gentle with the increase of the speed. In the fixed shallow-water depth, the total resistance of the vessel model will rise with the increase of the speed. And in the identical speed, the total resistance will lower with the increasingly deeper depth. Directly, we can obtain the resistance changing situation according to Fig.8, which infers that the shallow-water effect to the vessel model of the 
twin-skeg buoy tender should not be ignored. Further, in a certain speed, the resistance increasing percentage will touch the top firstly, afterwards gradually decrease.

\section{Conclusion}

Generally, the celerity performance of the inner-river vessel need to be predicted based on a series of resistance experiments for the shipbuilding and design. However, due to the obvious distinction of the experimental towing tank with the Heilongjiang River channel, in a relative extent, the shallow-water effect may be statistically ignored. In fact, in the real Heilongjiang channel, with the depth from 2 to 6 meters, the shallow-water effect must be set in an extremely significant position. Practically, the greater the real vessel resistance is, the more the main engine power will be than the reference value. Therefore, according to such researches above, it is proved that, the shallow-water effect must be indeed valued during the design of the inner-river ships of the Heilongjiang River channel. For the channel depth is an extremely important aspect, which will affect directly the celerity performance of the vessel.

\section{Reference}

[1] LI Shimo. Inland Twin-skeg Ship energy saving type [A]. China inland water transport ship good energy saving type assembly [Z], 1993.

[2] ZHANG Da-you, ZHU Tao, LI Shao-bo. Research and development analysis of twin skeg ship form [J].Ship Engineering. 2006, 28(6): 57-59. (in Chinese).

[3] ZHOU Xueyi. Hydraulic Calculation[M]. Beijing: Tsinghua University Press,1995.

[4] SHENG Zhenbang. Principles of Naval Architecture [M]. Shanghai: Shanghai Jiao Tong University Press,2003: 293-298.

[5] XU Nia.The Lines of Twin-Skeg Hull Design Method Research[D]. Dalian: Dalian University of Technology,2009.

[6] ZHANG Zhi-rong, ZHANG Feng, LI Bai-qi, Application of k-w turbulence model to computation of viscous flow field around a ship[J]. Journal of Ship Mechanics, 2003,7(1): 33-37. (in Chinese).

[7] Spalart P R, Allmaras S R. A one-equation turbulence model for aerodynamics flows. AIAA $92-0439$.

[8] ZHANG Jingzhou. Catamaran-stern Hull's Lines haracterand LocalTransformation Method Research[D]. Wuhan:WuhanUniversityof echnology,2007.

[9] Akhot V, Orzag S. A, Renormalization Group Analysis of Turbulence: Base Theory J. Scient Comput. 1986, (1): 3-11P.

[10] HUANG Li. Numerical Simulation of Viscous Flow around Three-Dimensional Hull by CFD [J]. Guangdong: Guangdong Shipbuilding,2011,30(3). 